\title{
Dense cold matter
}

\author{
Alexey Stavinskiy ${ }^{1,2, *}$ \\ ${ }^{1}$ National Research Center Kurchatov Institute, Institute for Experimental and Theoretical Physics, \\ 117218 Moscow, Russia \\ ${ }^{2}$ Joint Institute for Nuclear Research, 141980 Dubna, Russia
}

\begin{abstract}
Opportunities to study unexplored domains of nuclear matter phase diagram with BM@N and SPD-NICA is discussed. Since the optimal initial energy range depends on the phase diagram domain, the subject and experimental methods for its study have a lot of opportunities both for the fixed target and collider experiments. Fixed target experiments provide an access to the laboratory study of nuclear matter with the unprecedented density as high as the one for the neutron star core and even larger. A specialised kinematic trigger for relativistic ion-ion collisions can be used for this study. On the other hand, the NICA collider energy range will provide unique opportunities to study interactions between multi-quark states. The article discusses the importance of experimental study of the diquark component of nuclear structure function and diquark-diquark interactions. It is important to develop neutron identification methods both for the fixed target and collider experiments.
\end{abstract}

\section{Introduction}

Perturbative aspects of QCD have been tested up to a few percent. In contrast, nonperturbative aspects of QCD (hadronization, confinement, etc.) have been poorly tested. The study of the quark matter at different temperature and baryon density is part of efforts to consolidate the grand theory of particle physics. For that reason it is important to increase the phase diagram region accessible for experimental study at the laboratory. Traditional methods (heavy ion collisions at different initial energy and impact parameters) do not provide the access to extremely high baryon density domain of the phase diagram. A new approach to the laboratory study of extremely dense baryonic matter at low temperature has been offered by FLINT collaboration $[1,2]$. The key point of this proposal is a high $p_{t}$ cumulative trigger for this study. This trigger provides selecting interactions between the dense baryonic matter droplets (cumulative process), which can be considered as droplets of dense cold matter. New experiments in IPHE (SPIN) and JINR (SRC-BM@N) show the growing interest to the study of cumulative processes. It is evident, that modern detectors which are operating, being constructed and planned for the Nuclotron-NICA complex will open new opportunities in studying the dense nuclear matter and cumulative processes. For that reason it seems important to intensify discussions on perspectives of the dense cold matter study at the complex Nuclotron-NICA. These opportunities are discussed below. The new aspects of proposed measurements are related with the NICA-SPD project. A unique set of detectors, proposed

\footnotetext{
*e-mail: stavinsk@itep.ru
} 
for NICA-SPD can be used to study both high temperature and high baryonic density corner of the phase diagram.

\section{Experiments at Nuclotron}

The study of quark gluon plasma at extremely high initial energy have been discussed for a long period of time. The lifetime and volume of produced plasma increase with the initial energy. But high energy collisions are not appropriate to study the rich baryon states. That is the reason for new projects like FAIR and NICA. To study the dense cold matter, the optimal initial energy even smaller and some important results can be obtained at the Nuclotron energy range. The main tasks of this study could be structured as follows:

1) to study the properties of dense cold matter fluctuations ("fluctons" in terms of D.I. Blokhintsev [3]) in the ordinary nuclear matter;

2) to study interactions between fluctons;

3) to create as large as possible droplets of a new state of matter using interactions between fluctons.

Since the discovery of the nuclear cumulative effect in the early 1970-ies, the main efforts have been concentrated on the first task. It is well known that inclusive particle spectra in the target fragmentation region become independent on the initial energy starting from $E_{0} \sim 10 \mathrm{GeV} /$ nucleon. For this reason a lot of successful experiments in this field have been carried out at the Nuclotron (LPHE), U-10 (ITEP), CEBAF (TJNAF). A lot of important results have been obtained with relatively simple detectors (see, for example [4, 5]). But these data are restricted mainly by inclusive measurements of nucleon, pion, and kaon energy spectra. The data on strange baryon production in the cumulative process vary scarcely and are restricted by $\Lambda$ hyperons. Particle correlations have been studied in few experiments (see, for example [6]) and mainly for protons. It is evident that new generation experiments in this field could improve our knowledge about fluctons significantly. The accent would be done on strange particle production, correlation measurements(including femtoscopy). The last example of new generation experiments within the correlation approach is SRC-BM@N experiment at JINR (started at 2018, see M. Patsyuk report at this conference [7]).

As for the strange particle production in cumulative processes, it is important to include $\Sigma$ hyperons into analysis due to several reasons. The strangeness enhancement is well known properties of the cumulative process. The main components of strangeness in the energy range of the order of few $\mathrm{GeV}$ are $\mathrm{K}, \Lambda$, and $\Sigma$. That is because the threshold for kaonhyperon pair production is smaller than for the kaon-antikaon pair. The difference in the energy threshold is even more important for the cumulative process. But real experimental data are only for relatively stable $K^{ \pm}$and for $\Lambda$, which can be identified due to $\pi^{-}$p decay in track detectors. The data on $\Sigma$ hyperon production is needed not only "to complete the list of strange particles". The importance of electromagnetic and isotopic effects in the cumulative process is known for a long period of time [8]. The ratios $\Sigma^{+} / \Sigma^{-}, \Sigma^{0} / \Lambda$ provide unique information about these effects due to quark content(uus)/(dds) and (uds)/(uds), respectively.

As for the third task, possible way to produce droplets of cold and dense nuclear matter with the number of quarks up to $n_{q} \sim 20$, was proposed within FLINT experimental program in ITEP [1]. To produce these droplets, one needs a specialized trigger to select fluctonflucton interactions with a rare final state. This final state appears due to a coalescence mechanism from the quarks which belong both: to target and projectile fluctons. The cross section of the coalescence decreases strongly with the flucton relative momentum. For that reason the optimal initial energy for this study at the fixed target experiments is not larger than $E_{0} \sim 2-3 \mathrm{GeV} /$ nucleon. This is a perspective task for BM@N. Being produced the droplets of the cold and dense nuclear matter have to be extensively studied experimentally. Relative 
momentum distributions between the nucleons within the droplet, its space-time parameters measured by femtoscopy, isotopic properties do not complete a list of possible experiments. A theoretical support for this study is extremely important.

\section{Collider experiments}

The study of particle spectra and correlations in the fragmentation region provides information on properties of fluctons before interactions on, in other words, the flucton component of nuclear wave function. To study the interaction between fluctons (the second task), one needs to avoid the fragmentation region both for the target and projectile. It means that collider experiments are at the beam energy of the order of $E_{01}, E_{02} \sim 10 \mathrm{GeV} /$ nucleon, or fixed target experiments at the equivalent beam energy (of the order of $E_{0} \sim 100 \mathrm{GeV} /$ nucleon). This study can be done at U-70 (IPHE) or NICA-SPD. A special interest in this context is to study high $p_{t}$ central rapidity particle spectra and correlations. These measurements will provide access to a possible diquark component of the nuclear structure function and could be a unique source of information on the diquark properties and interactions. A detailed discussion of the diquark aspect and list of referencies can be found in S.Shimanskiy report at this conference [9]. Information about the diquark component is very important to study the dense and cold nuclear matter. This matter can not be assembled from identical fermions due to Pauli blocking. Diquark formation could be one of the possible ways for bosonization of this system. Due to this reason diquarks can be considered as candidates for the main constituent of dense and cold baryonic matter.

It is also interesting to study light nucleus central interactions with as high as possible degree of thermalization. It could be selected by a trigger for a small number of spectators and suppressed high $p_{t}$ component. Of course, these measurements could be realized only for the high luminosity light nucleus collisions. These measurements will provide information on the properties of nuclear matter at both high baryonic density and high temperature. There are no experimental data in this corner of the phase diagram. SPD detector complex looks like one of the best candidates for the proposed measurements. To select light nucleus collisions with a small number of spectators, one needs to identify not only the charged but also neutral spectators (neutrons).

\section{Conclusions}

Our brief review of possible experiments has shown, that the large potential of both the fixed target and collider experiments at the Nuclotron-NICA complex to study the dense nuclear matter. The dense nuclear matter (both cold and hot) is practically Terra incognita up to now except, may be, the data on cumulative processes and astrophysical phenomena. The study of dense nuclear matter promises a discovery of new phenomena and new states of matter.

It is difficult to get an access to this phase diagram domain without rare triggers. For this reason, the proposed experiments are not very simple because of small cross sections for the processes to be studied. Moreover, for some important measurements the existing experimental methods need to be improved. Both $\Sigma^{ \pm}$identification and nucleon identification within droplets of cold and dense nuclear matter have shown the importance of neutron identification. To select light nucleus collisions with a small number of spectators at SPD, it is necessary to identify neutrons. It seems the neutron identification methods for a wide energy range (from tenth $\mathrm{MeV}$ to several $\mathrm{GeV}$ ) have to be developed or improved. The optimal solution may be different for various neutron energy. Some possible solutions are discussed in $[10,11]$.

This work was supported by the Russian Science Foundation under grant 16-02-00637. 


\section{References}

[1] I.G. Alekseev et al., Phys.At.Nucl. 71, 1848 (2008). Phys.At.Nucl. 78, 936 (2015)

[2] A. Stavinskiy for the FLINT Collaboration, in Proceeding of WPCF-2010, BITP, Kiev, Physics of Particle and Nuclei Letters 8, 912 (2011)

[3] D.I. Blokhintsev, JETP 33, 1295 (1957)

[4] V.S. Stavinskiy, EPAN 10, 949 (1979)

[5] G.A. Lexin, Sov.J.Nucl.Phys. 65, 2042 (2002)

[6] Yu.D. Bayukov et al., Sov.J.Nucl.Phys. 50, 1023 (1989). Phys.Lett.B 189, 291 (1987)

[7] M. Patsyuk et al., report at XXIV International Baldin Seminar on High Energy Physics Problems "Relativistic Nuclear Physics and Quantum Chromodynamics", http://indico.jinr.ru/getFile.py/access?contribId=27

\&sessionId $=15 \&$ resId $=0$ \&materialId $=$ slides \& $\operatorname{confId}=433$

[8] A.M. Baldin et al., Preprint JINR-1-8858, Dubna (1975)

[9] S. Shimanskiy, report at XXIV International Baldin Seminar on High Energy Physics Problems "Relativistic Nuclear Physics and Quantum Chromodynamics", http://indico.jinr.ru/getFile.py/access?contribId=10

$\&$ sessionId $=15 \&$ resId $=0 \&$ materialId $=$ slides \& confId $=433$

[10] V.S. Goryachev et al., PTE 4, 20 (2016)

[11] F. Sefkow and F. Simon for CALICE collaboration, arXiv:1808.0928[phyysics.ins-det] (2018) 